OPEN ACCESS

Edited by:

Changiz Geula,

Northwestern University, United States

Reviewed by:

Fabrizio Piras,

Santa Lucia Foundation (IRCCS), Italy

Chen Liu,

Army Medical University, China

${ }^{*}$ Correspondence:

Shen Bo

shenbo13305722014@163.com

Received: 06 May 2020 Accepted: 11 November 2020 Published: 21 December 2020

Citation:

Zhou W, Shen B, Shen W-q, Chen H,

Zheng Y-f and Fei J-j (2020) Dysfunction of the Glymphatic System Might Be Related to Iron Deposition in the Normal Aging Brain.

Front. Aging Neurosci. 12:559603.

doi: 10.3389/fnagi.2020.559603

\section{Dysfunction of the Glymphatic System Might Be Related to Iron Deposition in the Normal Aging Brain}

\author{
Wei Zhou ${ }^{1}$, Bo Shen ${ }^{1 *}$, Wei-qiang Shen ${ }^{1}$, Hao Chen ${ }^{1}$, Yi-feng Zheng ${ }^{1}$ and Jing-jing Fei ${ }^{2}$ \\ ${ }^{1}$ Department of Radiology, Huzhou Central Hospital, Affiliated Central Hospital of Huzhou University, Affiliated Huzhou \\ Hospital, Zhejiang University School of Medicine, Huzhou, China, ${ }^{2}$ Department of Internal Medicine, Huzhou Central Hospital, \\ Affiliated Central Hospital of Huzhou University, Affiliated Huzhou Hospital, Zhejiang University School of Medicine, Huzhou, \\ China
}

Objective: The study aims to detect the potential relationship between iron deposition and the function of the glymphatic system in the normal aging brain.

Methods: We recruited 213 healthy participants. We evaluated the function of the glymphatic system using the index for diffusivity along the perivascular space (ALPSindex), assessed iron deposition on quantitative susceptibility mapping (QSM), and analyzed their relationship.

Results: The mean age of participants was $60.1 \pm 7.3$, and 107 (50.2\%) were female. The mean ALPS-index was $1.4 \pm 0.2$. The QSM values of the caudate nucleus, putamen, globus pallidus, thalamus, red nucleus, substantia nigra, and dentate nucleus were all related to the ALPS-index (all $P<0.001$ ).

Conclusions: The main finding of the current study is that the regional brain iron deposition was related to the function of the glymphatic system.

Advances in knowledge: We first evaluated the relationship between deposition of brain iron and the dysfunction of the glymphatic system.

Keywords: glymphatic system, iron deposition, aging, brain, MRI

\section{INTRODUCTION}

Iron is an electron facilitator and is involved in many brain functions, including oxygen transport, myelin production, electron transfer, and neurotransmitter synthesis (Hare et al., 2013). Both imaging and postmortem analyses have shown that the concentration of iron in the brain is not uniform. Previous studies have demonstrated that iron accumulates in the normal aging brain, which might damage precognitive function (Ramos et al., 2014; Gong et al., 2015). However, the exact mechanism of iron deposition in the aging brain remains unclear.

Recent work has led to the discovery of the "glymphatic system," which is a coined phrase that combines "gl" for glia cell with "lymphatic system" (Plog and Nedergaard, 2018; Rasmussen et al., 2018). Within the glymphatic system, cerebrospinal fluid enters the brain via peri-arterial spaces, passes into the interstitium via astrocytic aquaporin-4, and then drives the peri-venous drainage of interstitial fluid and its solute (Plog and Nedergaard, 2018; Rasmussen et al., 2018). Evidence suggests that the glymphatic system is an important fluid-clearance system in the brain (Bakker et al., 2016). Numerous neurological disorders have been found to be closely related to the dysfunction of the glymphatic system, including Alzheimer's disease and Parkinson's disease (Rasmussen et al., 2018; Zou et al., 2019). Evidence also revealed that iron deposition was one of the most important underlying mechanisms in Alzheimer's disease and Parkinson's disease 
(Liu et al., 2018; Chen et al., 2019). Some scholars also believe that the glymphatic system may be the major contributory factor to the deposition and clearance of iron in brain tissue (Wang et al., 2020), but evidence is still lacking.

Taoka et al. proposed a new method using diffusion tensor imaging analysis along the perivascular space (ALPS)-index to evaluate the glymphatic system using diffusion tensor imaging (DTI) and susceptibility weighted imaging (SWI) (Taoka et al., 2017). The authors evaluated water diffusivity along the right-to-left direction of the periventricular brain tissue, which matched the running direction of the deep medullary veins. Expected water diffusivity along the rightto-left direction could partially reflect the function of the glymphatic system. Using this method, they demonstrated a significant correlation between water diffusivity along the right-to-left direction and severity of cognitive dysfunction in Alzheimer's disease.

Therefore, in this study, we evaluated the function of the glymphatic system by ALPS-index based on DTI and SWI, assessed brain regional iron deposition using quantitative susceptibility mapping (QSM) based on SWI, and analyzed the relationship between them, aiming to discover the link between the glymphatic system and iron deposition in brain tissues.

\section{MATERIALS AND METHODS}

\section{Patients}

This study was approved by our institutional review board, and the requirement for patient consent was waived. From December 2017 to September 2019, 213 healthy participants (106 male and 107 female, age range 43-80) underwent multi-mode MRI scans.

\section{MRI Protocol}

MRI was performed on a 3.T scanner (GE Discovery MR750). The standard MRI protocol included axial unenhanced T1weighted imaging (T1WI) (TR, $500 \mathrm{~ms}$; TE, $10 \mathrm{~ms}$; slice thickness, $5 \mathrm{~mm}$; field of view, $240 \mathrm{~mm}$; matrix $=320 \times 356)$, DTI (b, 0; and b, 2,000 s/mm $\mathrm{mm}^{2}$, TR, 6,600 ms, TE, $89 \mathrm{~ms}$, MPG, 30 directions, field of view, $230 \mathrm{~mm}$, matrix $=94 \times 94$, slice thickness, $3 \mathrm{~mm}$ ), and SWI (TR, $22 \mathrm{~ms}$; TE, $11.5 \mathrm{~ms}$; field of view, $230 \mathrm{~mm}$; slice thickness, $2 \mathrm{~mm}$; matrix, $320 \times 251$; flip angle, $20^{\circ}$ ).

\section{Image Analysis}

The magnitude image and phase image were generated from the SWI raw image. Then the magnitude image was processed to generate a brain mask on a brain extraction tool contained in the FMRIB Software Library (Jenkinson et al., 2012). Phase images were divided by $2 \pi \mathrm{T}_{\mathrm{E}}$ to obtain a

TABLE 1 | Demographic and basic imaging data.

\begin{tabular}{lc}
\hline Variables & Mean \pm SD \\
\hline Age, year & $60.1 \pm 7.3$ \\
ALPS-index & $1.4 \pm 0.2$ \\
Caudate nucleus, $\times 10^{-3} \mathrm{ppm}$ & $79 \pm 25$ \\
Putamen, $\times 10^{-3} \mathrm{ppm}$ & $88 \pm 28$ \\
Globus pallidus, $\times 10^{-3} \mathrm{ppm}$ & $75 \pm 27$ \\
Thalamus, $\times 10^{-3} \mathrm{ppm}$ & $45 \pm 22$ \\
Red nucleus, $\times 10^{-3} \mathrm{ppm}$ & $79 \pm 27$ \\
Substantia nigra, $\times 10^{-3} \mathrm{ppm}$ & $66 \pm 24$ \\
Dentate nucleus, $\times 10^{-3} \mathrm{ppm}$ & $61 \pm 32$
\end{tabular}

ALPS-index: the index for diffusivity along the perivascular space.
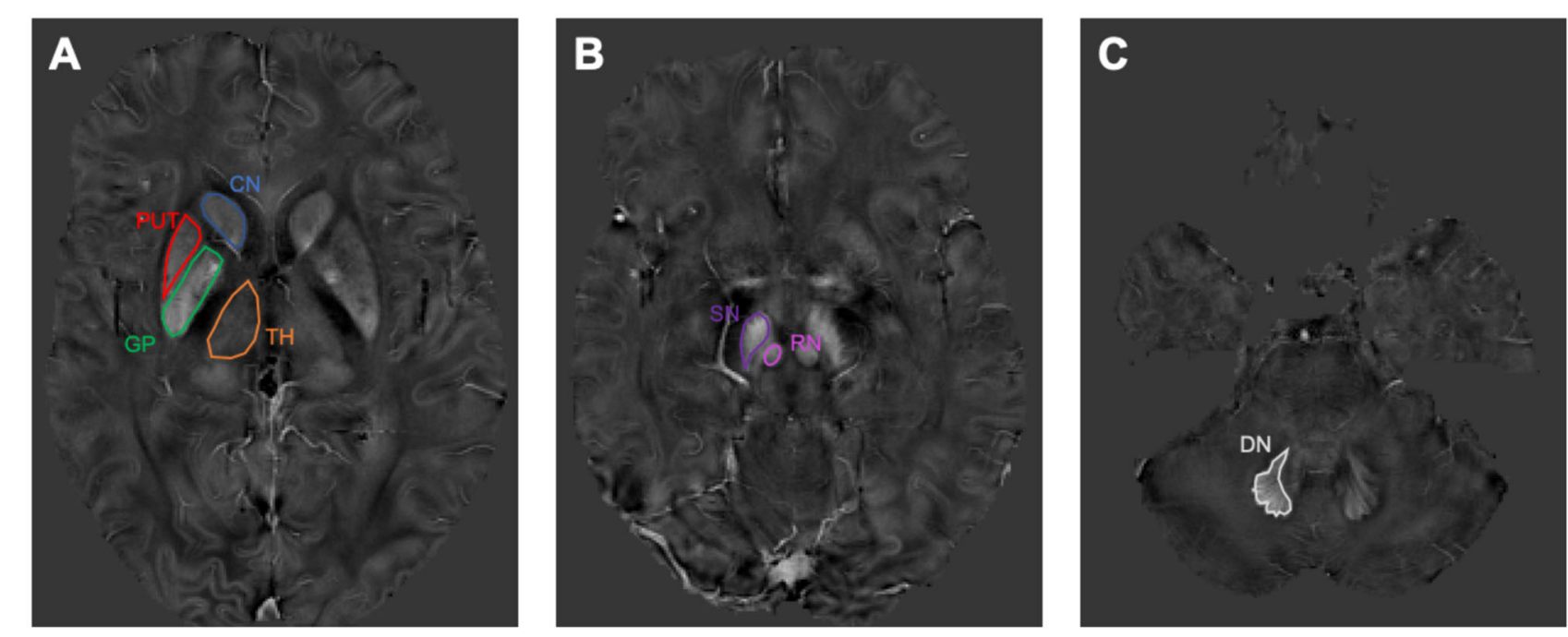

FIGURE 1 | The susceptibility maps illustrate the selected regions of interest covering the cerebrum, midbrain, and cerebellum. (A) shows the region of interests (ROIs) of caudate nucleus (CN), putamen (PUT), globus pallidus (GP), and thalamus (TH). (B) shows the ROls of red nucleus (RN) and substantia nigra (SN). (C) shows the ROI of dentate nucleus (DN). 
raw frequency map, and then the background field frequency was removed using the modified SHARP method (Schweser et al., 2011). Then the QSM mapping was derived by the LSQR method (Li et al., 2011). The regions of interest (ROIs) were determined by a previous publication (Guan et al., 2017). ITK-SNAP (www.itksnap.org) was used to perform manual segmentation and to measure the QSM values for each nucleus. Data for each region were obtained from the entire visible slice. ROIs were drawn to cover the caudate nucleus, putamen, globus pallidus, thalamus, red nucleus, substantia nigra, and dentate nucleus (Figure 1). An experienced neuroradiologist responsible for the ROI analysis was blinded to the information about the subjects, including disease status and demographics.

The ALPS-index was calculated as previously described by Taoka et al. (2017). On the color-coded fractional anisotropy map of the plane at the level of the lateral ventricle, 5-mm-diameter circle ROIs in the area of the projection fibers and in the area of the association fibers in the left hemisphere were performed by an experienced radiologist, who was blinded to the clinical data and other images. Then ALPS-index was calculated as ALPS index = mean (Dxproj + Dxassoc)/mean (Dyproj + Dzassoc).

We auto-segmented white matter hyperintensities using the LST toolbox and measured the volumes of white matter hyperintensities (Wirth et al., 2019). Central and cortical brain atrophy were categorized into three scores $(0=$ none, 1 $=$ modest, and $2=$ severe) as shown in a previous study (Sato et al., 2016).

TABLE 2 | The relationship between the index for diffusivity along the perivascular space (ALPS-index) and regional brain iron depositions.

\begin{tabular}{lc}
\hline & ALPS-index $(\mathbf{r}, \mathbf{P})$ \\
\hline Caudate nucleus, $\times 10^{-3} \mathrm{ppm}$ & $-0.289,<0.001$ \\
Putamen, $\times 10^{-3} \mathrm{ppm}$ & $-0.337,<0.001$ \\
Globus pallidus, $\times 10^{-3} \mathrm{ppm}$ & $-0.280,<0.001$ \\
Thalamus, $\times 10^{-3} \mathrm{ppm}$ & $-0.284,<0.001$ \\
Red nucleus, $\times 10^{-3} \mathrm{ppm}$ & $-0.307,<0.001$ \\
Substantia nigra, $\times 10^{-3} \mathrm{ppm}$ & $-0.267,<0.001$ \\
Dentate nucleus, $\times 10^{-3} \mathrm{ppm}$ & $-0.333,<0.001$
\end{tabular}

\section{Statistical Analysis}

Statistical analyses were performed using the SPSS software version 22. All metric and normally distributed variables were reported as mean \pm standard deviation. Categorical variables were presented as frequency (percentage). Correlate associations between variables were assessed by Spearman correlation analysis. Differences were considered statistically significant at $P<0.05$. Since the values of seven nucleus were measured, the statistically significant $P$-value for correlate associations of nucleus and ALPS-index should be $<0.007$, according to the basic tests of Bonferroni.

\section{RESULTS}

In this study, 213 patients were included. Table 1 shows the demographic data. The mean age was $60.1 \pm 7.3$, and 107 (50.2\%) were female. The mean ALPS-index was $1.4 \pm 0.2$. The mean volumes of white matter hyperintensities were $9.7 \pm 6.8$. The median scores of brain atrophy on the cortical and center were $1(1,2)$ and $1(1,2)$, respectively. Table 2 shows the relationship between the ALPS-index and regional brain iron depositions. The QSM values of the caudate nucleus, putamen, globus pallidus, thalamus, red nucleus, substantia nigra, and dentate nucleus were all related to the ALPS-index (all $P<0.05$ ). Moreover, age was related to both the ALPS-index $(r=-0.263, P<0.001)$ and regional QSM values (caudate nucleus: $r=0.218, P=0.001$; putamen: $r=0.373, P<0.001$; globus pallidus: $r=0.180, P=$ 0.009; thalamus: $r=0.164, P=0.016$; red nucleus: $r=0.182, P$ $=0.008$; substantia nigra: $r=0.199, P=0.004$; dentate nucleus: $r=0.213, P=0.002$ ). Table 3 shows the relationship between ALPS-index and regional brain iron depositions after adjusting for age, volumes of white matter hyperintensities, and scores of brain atrophy. Figure 2 shows the correlation between the index for diffusivity along the perivascular space (ALPS-index) and regional brain iron depositions.

\section{DISCUSSION}

The main finding of the current study is that regional iron deposition in brain tissues was related to the function of the glymphatic system in normal aging persons. Previously, the

TABLE 3 | Multiple factors analysis of the index for diffusivity along the perivascular space (ALPS-index).

\begin{tabular}{|c|c|c|c|}
\hline & $\begin{array}{l}\text { Age }(\beta, P) \text { (adjusting } \\
\text { for ALPS-index) }\end{array}$ & $\begin{array}{l}\text { ALPS-index ( } \beta, P) \\
\text { (adjusting for age) }\end{array}$ & $\begin{array}{l}\text { ALPS-index }(\beta, P) \text { (adjusting } \\
\text { for age, volumes of white } \\
\text { matter hyperintensities and } \\
\text { scores of brain atrophy) }\end{array}$ \\
\hline Caudate nucleus, $\times 10^{-3} \mathrm{ppm}$ & $-0.175,0.006$ & $-0.374,<0.001$ & $-0.380,<0.001$ \\
\hline Putamen, $\times 10^{-3} \mathrm{ppm}$ & $-0.146,0.028$ & $-0.354,<0.001$ & $-0.342,<0.001$ \\
\hline Globus pallidus, $\times 10^{-3} \mathrm{ppm}$ & $-0.198,0.003$ & $-0.301,<0.001$ & $-0.306,<0.001$ \\
\hline Thalamus, $\times 10^{-3} \mathrm{ppm}$ & $-0.200,0.002$ & $-0.339,<0.001$ & $-0.355,<0.001$ \\
\hline Red nucleus, $\times 10^{-3} \mathrm{ppm}$ & $-0.185,0.004$ & $-0.359,<0.001$ & $-0.372,<0.001$ \\
\hline Substantia nigra, $\times 10^{-3} \mathrm{ppm}$ & $-0.213,0.001$ & $-0.254,<0.001$ & $-0.260,<0.001$ \\
\hline Dentate nucleus, $\times 10^{-3} \mathrm{ppm}$ & $-0.200,0.002$ & $-0.343,<0.001$ & $-0.361,<0.001$ \\
\hline
\end{tabular}




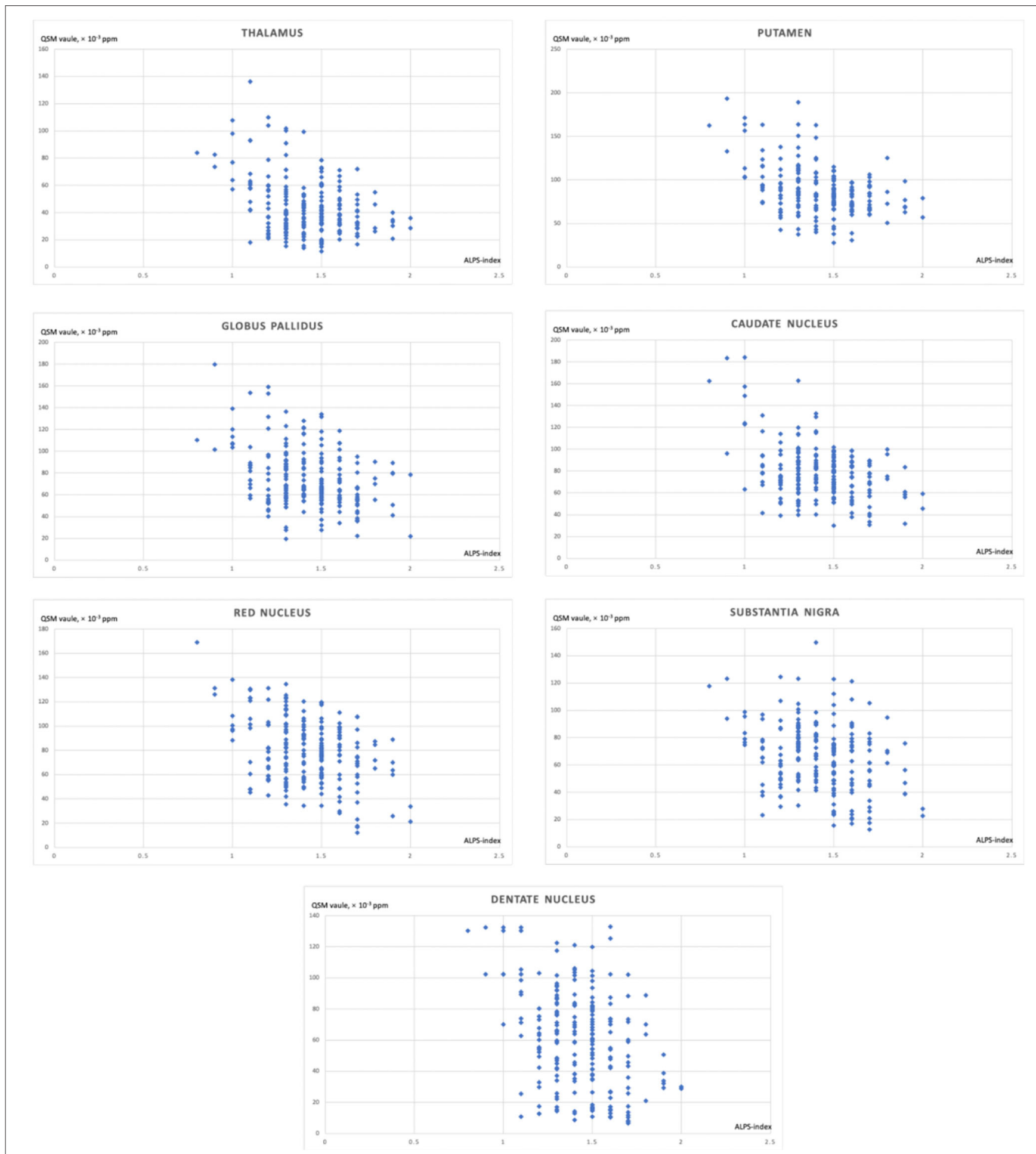

FIGURE 2 | The correlation between the index for diffusivity along the perivascular space (ALPS-index) and regional brain iron depositions.

glymphatic system has been speculated to be responsible for the clearance and homeostasis of waste in the brain (Bakker et al., 2016; Plog and Nedergaard, 2018; Rasmussen et al., 2018), but it remains unknown whether iron metabolism was related to the glymphatic system. Most recently, in animal models of intraventricular hemorrhage, the ability of iron drainage through deep cervical lymph nodes (DCLNs) was confirmed by Perl's Prussian blue reaction. They found that 
the DCLNs-excised group showed higher ferritin levels at areas surrounding ventricles than a DCLNs-preserved group 3 days after intraventricular hemorrhage (Wang et al., 2020). The result indicated the possible relationship between the function of the glymphatic system and iron drainage, since the DCLNs were recognized as downstream of the glymphatic system (Eide et al., 2018; Zhou et al., 2020). Our results support that in a healthy aging brain, the glymphatic system might also be involved in the clearance of iron, suggesting that iron metabolism shared the same pathway as other waste metabolisms. Moreover, a study has demonstrated that injury of the microvasculature and capillary-level microhemorrhages coincided with amyloid beta $(A \beta)$ deposits in senile plaques (Hansra et al., 2019). Iron deposition plays an important role in cerebral small vessel diseases (Del C Valdés Hernández et al., 2015). Therefore, we inferred that dysfunction of the glymphatic system might lead to the damage of microvasculature via deposition of $\mathrm{A} \beta$, then leading to iron deposition.

Moreover, iron deposition might also lead to the dysfunction of the glymphatic system. Iron metabolism mainly depends on iron regulatory proteins including ferritin, transferrin and transferrin receptor, hepcidin, ferroportin, and lactoferrin. A previous study had demonstrated that abnormal iron metabolism could generate hydroxyl radicals via the Fenton reaction, which could further trigger oxidative stress reactions, damage cell lipids, protein, and DNA structure and function, lead to cell death, and ultimately influence the process of $A \beta$ misfolding and plaque aggregation (Wang et al., 2019). The aggregation and deposition of $\mathrm{A} \beta$ in perivascular space may affect the bulk flow in perivascular space, directly leading to the dysfunction of the glymphatic system (Mestre et al., 2017). In addition, both iron deposition and dysfunction of the glymphatic system have been implicated in the pathogenesis of Alzheimer's Disease, Parkinson's Disease, and secondary injury following intracerebral hemorrhage, which supports the relationship between iron deposition and glymphatic function (Rasmussen et al., 2018; Farr and Xiong, 2020).

We also found a negative relationship between the function of the glymphatic system and age, which is in accordance with previous studies (Kress et al., 2014; Del C Valdés Hernández et al., 2015). Moreover, when both age and ALPS-index were set as independent variables, they were both independently related with regional QSM values. It might suggest that, although there are similar tends in increasing of iron deposition and decreasing of

\section{REFERENCES}

Bakker, E. N., Bacskai, B. J., Arbel-Ornath, M., Aldea, R., Bedussi, B., Morris, A. W., et al. (2016). Lymphatic clearance of the brain: perivascular, paravascular and significance for neurodegenerative diseases. Cell Mol. Neurobiol. 36, 181-194. doi: 10.1007/s10571-015-0273-8

Chen, Q., Chen, Y., Zhang, Y., Wang, F., Yu, H., Zhang, C., et al. (2019). Iron deposition in Parkinson's disease by quantitative susceptibility mapping. $B M C$ Neurosci. 20:23. doi: 10.1186/s12868-019-0505-9 glymphatic function with age, both of them have an independent effect on glymphatic dysfunction. Further studies are needed to clarify the mechanism.

An advantage of this study is that it is the first evaluation of the relationship between chronic deposition of iron and dysfunction of the glymphatic system. Our study also has several limitations. First, the subjects were restricted in our single center. Further multiple center studies with larger samples are needed. Second, the ROIs were placed manually, which may be a subjective factor of our measurement, although the radiologist is experienced and was blinded to clinical data and other images. Third, the method of the ALPS-index is theoretically deductive. Although it has demonstrated a relation with the severity of Alzheimer's disease and idiopathic normal pressure hydrocephalus, histological verification has not been performed.

\section{CONCLUSION}

The main finding of the current study is that regional brain iron deposition was related to the function of the glymphatic system.

\section{DATA AVAILABILITY STATEMENT}

The original contributions presented in the study are included in the article/supplementary material, further inquiries can be directed to the corresponding author/s.

\section{ETHICS STATEMENT}

The studies involving human participants were reviewed and approved by Huzhou Central Hospital Ethics Committee. The patients/participants provided their written informed consent to participate in this study.

\section{AUTHOR CONTRIBUTIONS}

BS designed the experiments. WZ carried out experiments. W-qS and $\mathrm{HC}$ analyzed experimental results. Y-fZ and J-jF participated in data collection. All authors wrote the manuscript.

\section{FUNDING}

Basic Public Welfare Research Project of Science and Technology Department of Zhejiang Province (LGF19H220001). The funding supported the collection of clinical and image data.

Del C Valdés Hernández, M., Ritchie, S., Glatz, A., Allerhand, M., Muñoz Maniega, S., Gow, A. J., et al. (2015). Brain iron deposits and lifespan cognitive ability. Age 37:100. doi: 10.1007/s11357-015-9837-2

Eide, P. K., Vatnehol, S. A. S., Emblem, K. E., and Ringstad, G. (2018). Magnetic resonance imaging provides evidence of glymphatic drainage from human brain to cervical lymph nodes. Sci. Rep. 8:7194. doi: 10.1038/s41598-018-25666-4

Farr, A. C., and Xiong, M. P. (2020). Challenges and opportunities of deferoxamine delivery for treatment of Alzheimer's disease, 
Parkinson's disease, and intracerebral hemorrhage. Mol. Pharm. doi: 10.1021/acs.molpharmaceut.0c00474. [Epub ahead of print].

Gong, N. J., Wong, C. S., Hui, E. S., Chan, C. C., and Leung, L. M. (2015). Hemisphere, gender and age-related effects on iron deposition in deep gray matter revealed by quantitative susceptibility mapping. NMR Biomed. 28, 1267-1274. doi: 10.1002/nbm.3366

Guan, X. J., Xuan, M., Gu, Q. Q., Huang, P. Y., Liu, C. L., Wang, N., et al. (2017). Regionally progressive accumulation of iron in Parkinson's disease as measured by quantitative susceptibility mapping. NMR Biomed. 30:10.1002/nbm.3489. doi: $10.1002 / \mathrm{nbm} .3489$

Hansra, G. K., Popov, G., Banaczek, P. O., Vogiatzis, M., Jegathees, T., Goldbury, C. S., et al. (2019). The neuritic plaque in Alzheimer's disease: perivascular degeneration of neuronal processes. Neurobiol. Aging 82, 88-101. doi: 10.1016/j.neurobiolaging.2019.06.009

Hare, D., Ayton, S., Bush, A., and Lei, P. (2013). A delicate balance: iron metabolism and diseases of the brain. Front Aging Neurosci. 5:34. doi: 10.3389/fnagi.2013.00034

Jenkinson, M., Beckmann, C. F., Behrens, T. E., Woolrich, M. W., and Smith, S. M. (2012). FSL. Neuroimage 62, 782-790. doi: 10.1016/j.neuroimage.2011.09.015

Kress, B. T., Iliff, J. J., Xia, M., Wang, M., Wei, H. S., Zeppenfeld, D., et al. (2014). Impairment of paravascular clearance pathways in the aging brain. Ann. Neurol. 76, 845-861. doi: 10.1002/ana.24271

Li, W., Wu, B., and Liu, C. (2011). Quantitative susceptibility mapping of human brain reflects spatial variation in tissue composition. Neuroimage 55, 1645-1656. doi: 10.1016/j.neuroimage.2010.11.088

Liu, J. L., Fan, Y. G., Yang, Z. S., Wang, Z. Y., and Guo, C. (2018). Iron and Alzheimer's disease: from pathogenesis to therapeutic implications. Front. Neurosci. 12:632. doi: 10.3389/fnins.2018.00632

Mestre, H., Kostrikov, S., Mehta, R. I., and Nedergaard, M. (2017). Perivascular spaces, glymphatic dysfunction, and small vessel disease. Clin. Sci. 131, 2257-2274. doi: 10.1042/CS20160381

Plog, B. A., and Nedergaard, M. (2018). The glymphatic system in central nervous system health and disease: past, present, and future. Annu. Rev. Pathol. 13:379-394. doi: 10.1146/annurev-pathol-051217111018

Ramos, P., Santos, A., Pinto, N. R., Mendes, R., Magalhães, T., and Almeida, A. (2014). Iron levels in the human brain: a post-mortem study of anatomical region differences and age-related changes. J. Trace Elem. Med. Biol. 28, 13-17. doi: 10.1016/j.jtemb.2013.08.001

Rasmussen, M. K., Mestre, H., and Nedergaard, M. (2018). The glymphatic pathway in neurological disorders. Lancet Neurol. 17, 1016-1024. doi: $10.1016 /$ S1474-4422(18)30318-1
Sato, S., Delcourt, C., Heeley, E., Arima, H., Zhang, S., Al-Shahi Salman, R., et al. (2016). Significance of cerebral small-vessel disease in acute intracerebral hemorrhage. Stroke 47, 701-707. doi: 10.1161/STROKEAHA.115.012147

Schweser, F., Deistung, A., Lehr, B. W., and Reichenbach, J. R. (2011). Quantitative imaging of intrinsic magnetic tissue properties using MRI signal phase: an approach to in vivo brain iron metabolism? Neuroimage 54, 2789-2807. doi: 10.1016/j.neuroimage.2010.10.070

Taoka, T., Masutani, Y., Kawai, H., Nakane, T., Matsuoka, K., Yasuno, F., et al. (2017). Evaluation of glymphatic system activity with the diffusion MR technique: diffusion tensor image analysis along the perivascular space (DTI-ALPS) in Alzheimer's disease cases. Jpn. J. Radiol. 35, 172-178. doi: $10.1007 / \mathrm{s} 11604-017-0617-\mathrm{z}$

Wang, B. C., Liang, C. F., Chen, C., He, H. Y., Huang, T. C., Gao, Q., et al. (2020). Intracranial lymphatic drainage discharges iron from the ventricles and reduce the occurrence of chronic hydrocephalus after intraventricular hemorrhage in rats. Int. J. Neurosci. 130, 130-135. doi: 10.1080/00207454.2019.1667780

Wang, T., Xu, S. F., Fan, Y. G., Li, L. B., and Guo, C. (2019). Iron pathophysiology in Alzheimer's diseases. Adv. Exp. Med. Biol. 1173, 67-104. doi: 10.1007/978-981-13-9589-5_5

Wirth, A. M., Johannesen, S., Khomenko, A., Baldaranov, D., Bruun, T. H., Wendl, C., et al. (2019). Value of fluid-attenuated inversion recovery MRI data analyzed by the lesion segmentation toolbox in amyotrophic lateral sclerosis. J. Magn. Reson. Imaging 50, 552-559. doi: 10.1002/jmri.26577

Zhou, Y., Cai, J., Zhang, W., Gong, X., Yan, S., Zhang, K., et al. (2020). Impairment of the glymphatic pathway and putative meningeal lymphatic vessels in the aging human. Ann. Neurol. 87, 357-369. doi: 10.1002/ana.25670

Zou, W., Pu, T., Feng, W., Lu, M., Zheng, Y., Du, R., et al. (2019). Blocking meningeal lymphatic drainage aggravates Parkinson's disease-like pathology in mice overexpressing mutated $\alpha$-synuclein. Transl. Neurodegener. 8:7. doi: 10.1186/s40035-019-0147-y

Conflict of Interest: The authors declare that the research was conducted in the absence of any commercial or financial relationships that could be construed as a potential conflict of interest.

Copyright $\odot 2020$ Zhou, Shen, Shen, Chen, Zheng and Fei. This is an open-access article distributed under the terms of the Creative Commons Attribution License (CC $B Y)$. The use, distribution or reproduction in other forums is permitted, provided the original author(s) and the copyright owner(s) are credited and that the original publication in this journal is cited, in accordance with accepted academic practice. No use, distribution or reproduction is permitted which does not comply with these terms. 